\title{
GENERALIZED STIELTJES CONSTANTS AND INTEGRALS INVOLVING THE LOG-LOG FUNCTION: KUMMER'S THEOREM IN ACTION
}

\author{
OMRAN KOUBA
}

Abstract. In this note, we recall Kummer's Fourier series expansion of the 1-periodic function that coincides with the logarithm of the Gamma function on the unit interval $(0,1)$, and we use it to find closed forms for some numerical series related to the generalized Stieltjes constants, and some integrals involving the function $x \mapsto \ln \ln (1 / x)$.

Mathematics subject classification (2010): 33B15.

Keywords and phrases: Gamma function, log-log integrals, Fourier series, numerical series.

\section{REFERENCES}

[1] M. Abramowitz And I. A. Stegan (Eds), it Handbook of Mathematical Functions, with Formulas, Graphs, and Mathematical Tables, Dover Books on Mathematics, Dover Publication, Inc., New York, 1972.

[2] V. AdamchiK, A class of Logarithmic Integrals, Proceedings of the 1997 International Symposium on Symbolic and Algebraic Computation, ACM, Academic Press, (2001), 1-8.

[3] M. Albano, T. Amdeberhan, E. Beyerstedt and V. H. Moll, The integrals in Gradshteyn and Ryzhik. Part 19: The error function, Scientia, 21, (2011), 25-42.

[4] G. E. Andrews, R. Askey AND R. Roy, Special functions, Encyclopedia of Mathematics and Its Applications, 71, Cambridge University Press, 1999.

[5] I. V. BLagouchine, A theorem for the closed-form evaluation of the first generalized Stieltjes constant at rational arguments and some related summations, Journal of Number Theory (Elsevier), 148, 537-592, (2015).

[6] D. Bierens DE HaAn, Nouvelles tables d'intégrales définies, Amsterdam, 1867, (reprint) G. E. Stechert \& Co., New York, 1939.

[7] I. S. Gradshteyn And I. M. Ryzhik, Table of Integrals, Series, and Products, 8th edition, D. Zwillinger, and V. Moll, eds. Academic Press, Elsevier Inc, 2015.

[8] E. Kummer, Beitrag zur Theorie der Function $\Gamma(x)$, J. Reine Ang. Math., 35, (1847), 1-4.

[9] V. H. MolL, The integrals in Gradshteyn and Ryzhik. Part 4: The gamma function, Scientia, 15, (2007), 37-46.

[10] V. H. MoLL, The integrals in Gradshteyn and Ryzhik. Part 6: The beta function, Scientia, 16, (2008), 9-24.

[11] L. A. Medina And V. H. Moll, A Class of Logarithmic Integrals, Ramanujan Journal, 20, (2009), 91-126.

[12] V. H. MoLL, Special Integrals of Gradshteyn and Ryzhik: the Proofs - Volume I, CRC Press, Taylor \& Francis Group, LLC, 2015.

[13] V. H. MoLL, Special Integrals of Gradshteyn and Ryzhik: the Proofs - Volume II, CRC Press, Taylor \& Francis Group, LLC, 2016.

[14] I. VARDI, Integrals, an Introduction to Analytic Number Theory, The American Mathematical Monthly, 95, no. 4, (1988), 308-315.

[15] Wolfram Research, Inc. Mathematica, Version 11.0, Wolfram Research, Inc. Champaign, Illinois, 2016. 